\title{
Caracterización de bordes de bosque altoandino e implicaciones para la restauración ecológica en la Reserva Forestal de Cogua (Colombia)
}

\author{
Alba Lucía Montenegro \& Orlando Vargas Ríos \\ Departamento de Biología, Universidad Nacional de Colombia, A.A. 14490 Bogotá, Colombia, Fax: 3165310; mon- \\ tenegro.alba@gmail.com, jovargasr@gmail.com
}

Recibido 30-I-2007. Corregido 30-VI-2008. Aceptado 31-VII-2008.

\begin{abstract}
Characterization of High Andean forest edges and implications for their ecological restoration (Colombia). The growth of a forest patch through colonization of the adjacent matrix is mostly determined by the particular characteristics of the edge zone. Knowing how these characteristics are related to a specific edge type and how they influence the regeneration process, is important for High Andean forest edges restoration. This study aimed to characterize three types of High Andean forest edge in Cogua Forest Reserve (Colombia): 1) edge of Chusquea scandens, 2) "paramizado", and 3) old edge, characterized for being in a later successional state. Two forest patches were chosen for each edge type and 13 criteria were analyzed; these were of topographic order, micro-environmental order, vegetation structure and species composition. In each patch the vegetation was evaluated by means of two $60 \mathrm{~m}$ transects perpendicular to the edge and along the matrix-edgeinterior of the forest gradient. All woody plant species were identified and counted to determine their abundance. Environmental variables (air temperature, relative humidity, wind speed, and light radiation) were measured in one of the transects. Three of the 13 criteria were of little importance in shaping the type of edge habitat (slope, patch shape and area). The others were closely related with the micro-environmental conditions and in turn with the vegetation structure and composition; this relationship confers particular characteristics to each edge type. The microclimate and floristic edge limits coincided; edges extend between 10 and $20 \mathrm{~m}$ into the forest depending on the edge type. The paramizado edge has the smallest environmental self-regulation capacity and is more exposed to fluctuations of the studied variables, because of its greatest exposition to the wind action and loss of the tallest trees (between 10 and $15 \mathrm{~m}$ ) which regulate the understorey microclimate. This low environmental buffer capacity prevents the establishing of mature forest species (for example, Schefflera sp. and Oreopanax bogotensis) although they are found in other areas within the same patch. All these results show that the paramizado edge needs the most intervention for its restoration. The Chusquea scandens edge forest is the most sheltered since this species acts as a protecting shield. However it still needs to be controlled to allow the adjacent matrix colonization by the forest species and natural regeneration, as it does in the old edge type forest, which moreover has an intermediate self-regulating capacity relative to the other two. The vegetation composition reveals that most of the edge species can also grow inside, beyond the forest edge. Rev. Biol. Trop. 56 (3): 1543-1556. Epub 2008 September 30.
\end{abstract}

Key words: Chusquea scandens, disturbance, ecological restoration, forest edge, matrix, microclimate.

Uno de los aspectos derivados de la fragmentación es la creación de zonas de borde en el límite entre los parches de bosque y la matriz circundante. El predominio de los ambientes de borde, generados por eventos de disturbio entre comunidades de vegetación adyacentes, determina fuertemente la expresión de los procesos ecológicos en los paisajes fragmentados.
Esto se debe a que los bordes pueden controlar cambios espacio temporales en las condiciones microclimáticas, así como la distribución y disponibilidad de recursos requeridos por las plantas para establecerse y crecer, ejerciendo un control significativo sobre el crecimiento de las plántulas y los patrones de supervivencia a escala del paisaje. La capacidad de 
regeneración de un fragmento de bosque depende de las características particulares, tanto del parche mismo como de su zona de borde. Un fragmento puede presentar diferentes tipos de borde simultáneamente, debido a variaciones en estructura, composición florística, fisonomía (Williams-Linera 1990, Williams-Linera et al. 1998, Didham y Lawton 1999, Arango 2002), ubicación, topografía, área del parche (Primack 1993, Murcia 1995) tipo de matriz, régimen de disturbio (Murcia 1995, Fox et al. 1997, Mesquita et al. 1999, Buckley 2000, Williams-Linera et al. 2002) y microclima (Williams-Linera et al. 1998, Harper et al. 2005), entre otros. Este último está bastante determinado por la interacción de los demás factores que en conjunto definen el tipo de hábitat de borde resultante, su capacidad para amortiguar las fluctuaciones microclimáticas extremas de la matriz circundante, su resiliencia siguiendo un disturbio (Absjornsen et al. 2004) y su capacidad para favorecer o retrasar la regeneración y el avance del bosque hacia la matriz (Williams-Linera 1990, Williams-Linera et al. 1998, Harper et al. 2005). Por lo anterior, para lograr el éxito en la restauración del bosque es necesario considerar las características particulares de los bordes, ya que pueden facilitar el proceso o impedirlo cuando mantienen activas tendencias degradativas en el parche en cuestión o impiden el desarrollo de las especies del bosque. Asimismo, diferentes tipos de borde requieren estrategias de restauración diferentes, en aras a favorecer la colonización de la matriz por parte de las especies del bosque y la expansión del mismo. Este trabajo pretende establecer los factores fisiográficos, microclimáticos y de la vegetación determinantes en el tipo de hábitat de borde, mediante la comparación de tres tipos de borde (antiguo, paramizado y de chusque) así como establecer sus limitaciones para la regeneración del bosque, respondiendo a las siguientes preguntas: ¿Qué factores son más importantes en la formación de un determinado tipo de borde? y ¿Qué limitantes para la regeneración del bosque presenta cada tipo de borde?

\section{MATERIALES Y MÉTODOS}

Sitio de estudio: La Reserva Forestal Municipal de Cogua (RFMC) está ubicada a los $5^{\circ} 4^{\prime} 35^{\prime \prime} \mathrm{N}$ y $74^{\circ} 00^{\prime} 00^{\prime \prime} \mathrm{W}$, presenta una escala altitudinal entre los 2970 y $3650 \mathrm{~m}$, una temperatura media anual de $10.5^{\circ} \mathrm{C}$, un régimen de lluvias bimodal y un promedio anual de precipitación de $1046 \mathrm{~mm}$. Se localiza en la vereda Quebradahonda, sobre el flanco oriental del Páramo de Guerrero; tiene una superficie de 500 ha (Zambrano 1995). La vegetación de la Reserva es el resultado de la historia de disturbios producidos antes de la creación de la misma; el análisis de este aspecto se fundamenta en la deducción basada en las observaciones de campo, la estructura actual de la vegetación e informacion secundaria, (León 2002, García y Giraldo 2003, Díaz 2004). El bosque estuvo sometido a la fragmentación por diversas actividades agropecuarias, por lo cual pueden encontrarse parches de bosque en diferente estado de regeneración dependiendo del régimen de disturbio y del tiempo de abandono transcurrido desde su vinculación a la misma. Los parches con bordes antiguos llevan más tiempo de abandono (14 años) y la regeneración se encuentra en un estado más avanzado, mientras los parches con bordes paramizados o de chusque presentan tiempos de abandono entre seis y nueve años (Díaz 2004) y estados de regeneración menos avanzados. El régimen de disturbios de las matrices adyacentes, conduce en mayor o menor grado a una vegetación de porte herbáceo altamente contrastante con el bosque, no sólo a nivel estructural sino también ambiental; las matrices que limitan con los bordes paramizados se encuentran en zonas de difícil acceso y pendientes pronunciadas o cercanas a escarpes lo que posiblemente impidió su explotación directa, pero no su alteración como resultado de la creación de trochas de acceso para la extracción de madera de los parches con este tipo de borde; esto unido a las condiciones ambientales desfavorables, por la exposición directa al viento, generó una matriz mixta en especies de páramo y subpáramo. Las matrices que limitan con los bordes antiguo o de chusque 
fueron sometidas a un grado de disturbio medio (quema-pastoreo) o alto (quema-cultivo-pastoreo). Ambos bordes fueron clareados con el fin de aumentar el área útil para estas actividades, además de brindar sombra al ganado. Este clareo así como la extracción selectiva de madera aún son evidentes en la estructura del bosque, debido a que la expansión lateral de las copas de los árboles remanentes no alcanzó a cerrar los claros totalmente. Por su parte, los bordes paramizados poseen trochas que conectan con caminos principales, las cuales seguramente fueron utilizadas para la extracción de madera intensivamente, como revela la mayor amplitud del clareo y la ausencia de los árboles más altos (entre 8 y $10 \mathrm{~m}$ ) que sí se encuentran en los otros tipos de borde.

Clasificación de los bordes: Se realizó el reconocimiento de los bordes presentes en la zona de estudio, para clasificarlos mediante inspección directa, con base en 13 criterios (Cuadro 1) de acuerdo con Murcia (1995). Se decidió considerar solamente aquellos parches de bosque con un tamaño superior a $5000 \mathrm{~m}^{2}$, porque su vegetación tiene más de dos estratos de árboles e individuos con más de $7 \mathrm{~m}$ de altura cuyas copas conforman un dosel; además, esta área resultó ser suficiente para generar un microclima interior. Los parches con estas características son poco numerosos en la Reserva y solamente presentan los tres tipos de borde seleccionados: $a$. Bordes de chusque, b. Bordes paramizados, c. Bordes antiguos.

Muestreo: Considerando la escasez de los fragmentos relativamente grandes en la RFMC, sólo fue posible escoger dos parches de bosque para cada tipo de borde. En cada parche se realizaron dos transectos de igual longitud, separados por una distancia de $10 \mathrm{~m}$. Cada transecto tuvo una longitud de $60 \mathrm{~m} \mathrm{y}$ se abarcaron $2 \mathrm{~m}$ a cada lado del mismo. Las cuerdas empleadas se marcaron cada $10 \mathrm{~m}$ para facilitar el análisis y se colocaron perpendiculares al borde de interés, abarcando el gradiente matriz-borde-interior del bosque, dejando $20 \mathrm{~m}$ en la matriz y los $40 \mathrm{~m}$ restantes dentro del bos- que (Arango 2002). Se identificaron y contaron todos los individuos leñosos con más de $30 \mathrm{~cm}$ de altura, presentes en esa área. Las especies presentes en la Reserva se identificaron en estudios previos realizados en la misma (Díaz 2004, Trujillo 2004), cotejadas con ejemplares botánicos del Herbario Nacional Colombiano (COL). Adicionalmente, en cada tipo de borde se realizaron mediciones de temperatura, humedad ambiental, velocidad del viento y radiación lumínica, durante dos días consecutivos, según lo planteado por Williams-Linera et al. 1998. Los datos se tomaron tres veces en el día (8, 13 y 17 horas) a lo largo de uno de los dos transectos de cada parche de bosque, en los puntos marcados: matriz, $0 \mathrm{~m}, 10 \mathrm{~m}, 20 \mathrm{~m}, 30$ $\mathrm{m}$ y $40 \mathrm{~m}$.

Análisis de datos: Para establecer la amplitud del borde con base en la composición de especies, que tiende a ser diferente entre esta zona y el interior del bosque, se realizó un Análisis de Correspondencia (AC) con el programa PAST versión 1.40 (Hammer et al. 2001) actualización enero de 2006. También se realizó el ANOVA no paramétrico, dejando como factores el tipo de borde y la distancia en el transecto, con el programa PERMANOVA v. 1.6 (Anderson 2001).

\section{RESULTADOS}

Detección del borde: El análisis de correspondencia (Fig. 1) detecta una zona de borde que abarca $10 \mathrm{~m}$ en el borde antiguo y $20 \mathrm{~m}$ en los bordes de chusque y paramizado. El ANOVA realizado con PERMANOVA v. 1.6 detecta diferencias significativas entre los tres tipos de borde $(\mathrm{F}=3.6446, \mathrm{gl}=2, \mathrm{P}<0.005) \mathrm{y}$ diferencias en los sectores del transecto con la distancia $(\mathrm{F}=1.5042, \mathrm{gl}=12, \mathrm{P}<0.05)$ pero en las comparaciones posteriores no detecta hasta dónde va la zona de borde, porque no distingue cuáles sectores del transecto difieren entre sí (Anexo 1).

Caracterización de los hábitats de borde: De los 13 criterios seleccionados para 
CUADRO 1

Resumen de las características evaluadas en cada tipo de borde

TABLE 1

Synthesis of the characteristics evaluated in each edge type

Características

1. Orientación

2. Altitud

3. Área del parche

4. Forma del parche

5. Ubicación topográfica

6. Pendiente

7. Amplitud del borde

8. Estructura

9. Especies más abundantes

10. Fisonomía sotobosque

11. Microambiente

12. Matriz

13. Disturbio, regeneración
Borde de chusque

Sotavento NW, SW

Bajo: 3260 m, 3300 m

2.0 ha y 6.0 ha

Trapezoidal o triangular

En ladera

Entre $17^{\circ}$ y $30^{\circ}$

Entre $10 \mathrm{~m}$ y $20 \mathrm{~m}$

Compleja. Árboles de hasta $10 \mathrm{~m}$ de altura en el borde y

$15 \mathrm{~m}$ en interior

Chusquea scandens, Tibouchina grossa, Miconia ligustrina

Sotobosque denso

Potegido, cálido y húmedo. Autorregulación.

Principalmente herbácea (pastizal Holcus lanatus)

Moderada extracción de madera, claros medianos en el dosel.
Borde antiguo

Sotavento E, SW

Alto: $3380 \mathrm{~m}, 3420 \mathrm{~m}$

4.0 ha y 5.0 ha

Rectangular o triangular alargado

En ladera

Entre $15^{\circ}$ y $33^{\circ}$

$10 \mathrm{~m}$

Compleja. Árboles de hasta $10 \mathrm{~m}$ de altura en el borde y $15 \mathrm{~m}$ en interior

Tibouchina grossa, Gaiadendron punctatum, Miconia squamulosa

Sotobosque despejado

Condiciones intermedias. Autorregulación.

Herbácea con parches de arbustos del bosque que la están colonizando.

Moderada extracción de madera, claros medianos en el dosel.
Borde paramizado

Barlovento SE

Alto: $3380 \mathrm{~m}, 3400 \mathrm{~m}$

0.6 ha y 5.0 ha

Poligonal-circular o

rectangular

En la cima

Entre $8^{\circ}$ y $33^{\circ}$

Entre $10 \mathrm{~m}$ y $20 \mathrm{~m}$

Simplificada. Árboles de hasta $8 \mathrm{~m}$ de altura en el borde y $10 \mathrm{~m}$ en interior

Gaiadendron punctatum, Macleania rupestris, Tibouchina grossa

Sotobosque muy denso

Extremo, frío, seco. No autorregulación.

Herbácea con arbustos dispersos. Colonización avanzada de especies de páramo y subpáramo.

Alta extracción de madera, claros amplios en el dosel, muchos árboles caídos. caracterizar los tipos de borde, diez están estrechamente relacionados con éstos: estructura, composición, fisonomía, condiciones ambientales, disturbio, orientación, ubicación topográfica, altitud, amplitud del borde y tipo de matriz. Los tres atributos restantes (pendiente, forma y área del parche) no parecen ser tan relevantes, puesto que parches con un mismo tipo de borde presentan una amplia variación en estos criterios. Los resultados generales de las variables evaluadas en cada tipo de borde se presentan en la figura 2 y Cuadro 1.

\section{Descripción de los tipos de borde y com- posición de especies: \\ 1) Borde de chusque: La presencia de $C$. scandens es muy marcada y se reduce gra- dualmente hacia el interior, abarcando entre 8}




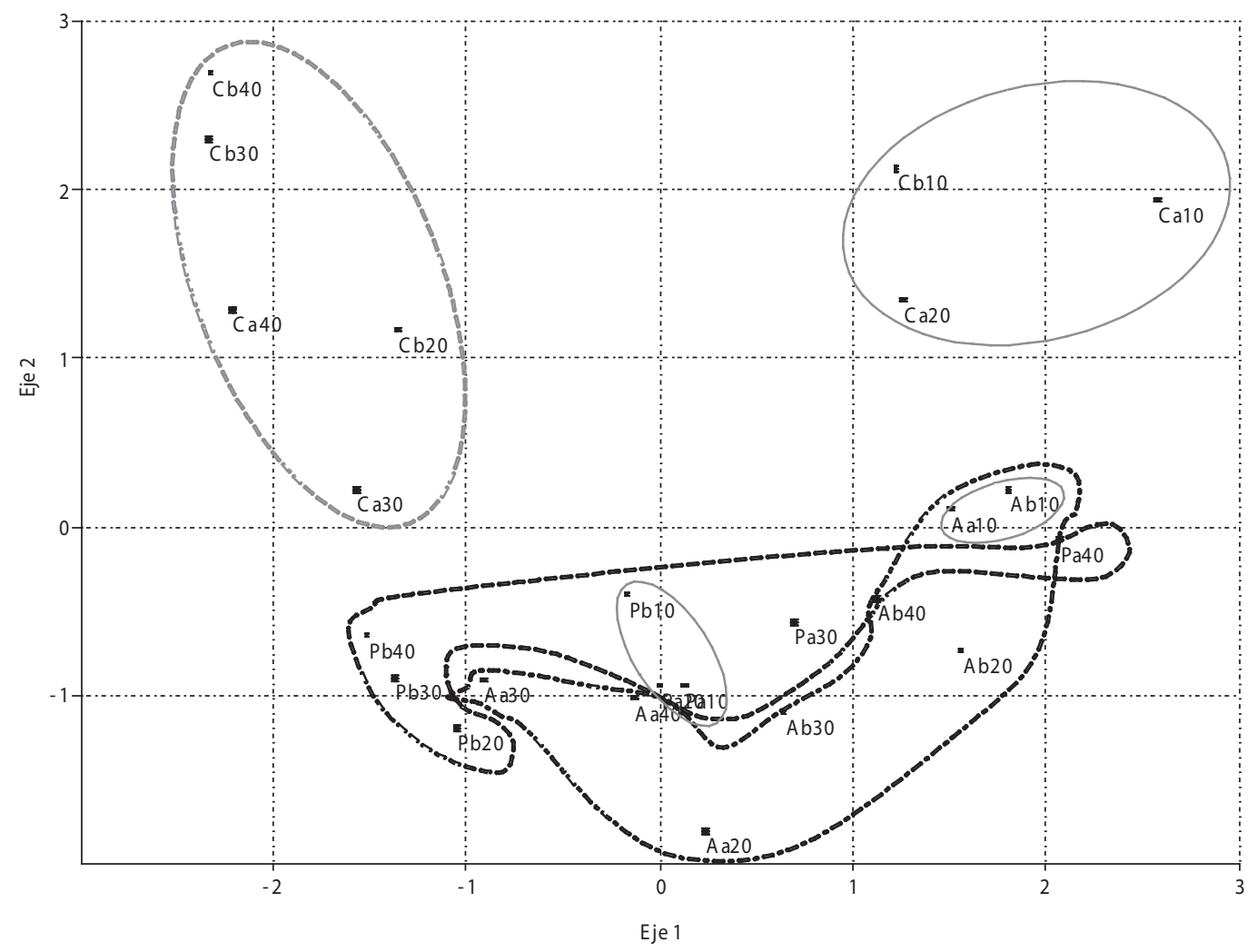

Fig. 1. Análisis de correspondencia para los sectores de los transectos realizados en el bosque.La letra mayúscula indica el tipo de borde (A: antiguo, C: chusque, P: paramizado); la letra minúscula indica uno de los dos parches de muestreo (a, b) y el número indica el sector del transecto en el bosque empezando en el límite con la matriz y finalizando en el interior (10: 0-10 m, 20: 10,1-20 m, 30: 20,1-30 m, 40: 30,1-40 m). La línea punteada rodea la zona interior del bosque en cada tipo de borde, la línea entera agrupa las zonas de borde.

Fig. 1. Correspondence analysis for the sectors of transects into the forest. Edge type (A: old edge, C: chusque edge, P: paramizado edge). Forest patches of each edge type $(a, b)$. Sectors of transect, in order from de matrix until the forest interior (10: 0-10 m, 20: 10,1-20 m, 30: 20,1-30 m, 40:30,1-40 m). Dotted line represents the forest interior zone in each edge type, whole line represents the edge zone.

y $12 \mathrm{~m}$, siendo las especies más abundantes Tibouchina grossa y Miconia ligustrina. El sotobosque es denso debido a la presencia del chusque. La matriz es altamente contrastante en su aspecto, al ser principalmente herbácea (pastizales de Holcus lanatus), se presentan arbolitos colonizadores de las especies mencionadas, pero el chusque tiende a crecer sobre ellos sofocándolos e impidiendo la expansión del bosque. Este tipo de borde se ubica en sotavento y la presencia del chusque actúa como una barrera protectora, por lo que es el borde más cálido y húmedo de los tres investigados (Fig. 3, Anexo 2). La vegetación del borde de chusque posee la capacidad de amortiguar las condiciones extremas de la matriz circundante.

2) Borde antiguo: Se ubica en los parches de bosque que llevan más tiempo en regeneración (14 años), lo cual se evidencia en la altura de los árboles y en el sotobosque despejado, pues la presencia de especies de subpáramo, de chusque y pioneras arbustivas es baja. Presenta una mayor abundancia de especies escasas en los demás parches, como Hedyosmum 


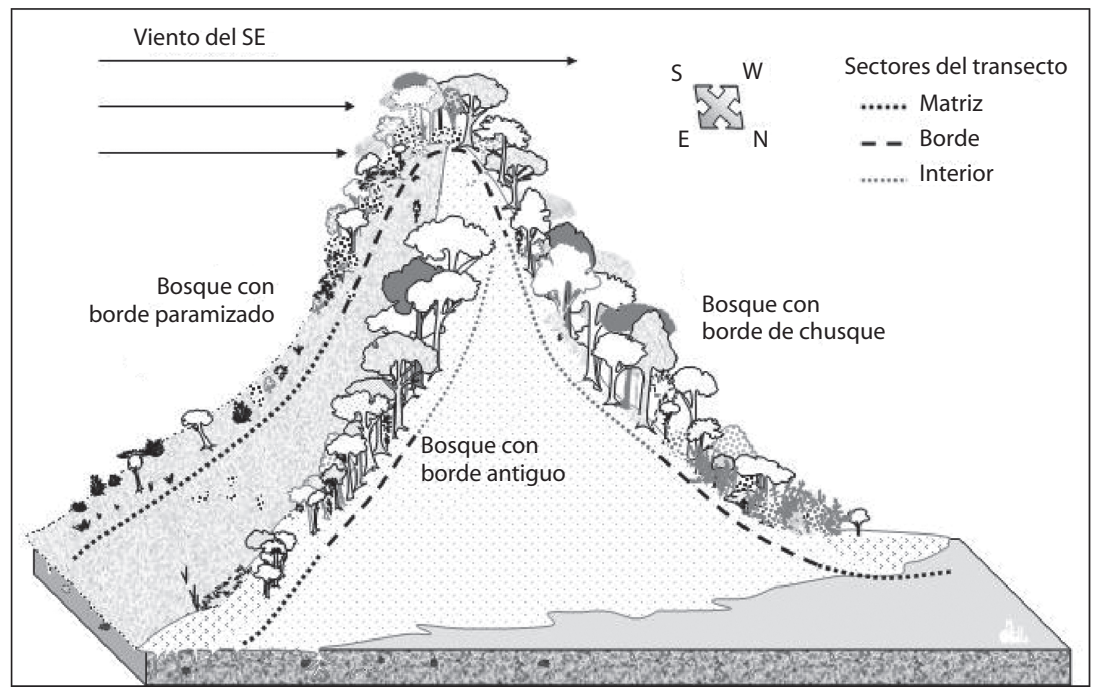

Fig. 2. Perfiles de la vegetación y resumen de las características de cada tipo de borde.

Fig. 2. Vegetation side view and synthesis of the characteristics evaluated in each edge type.
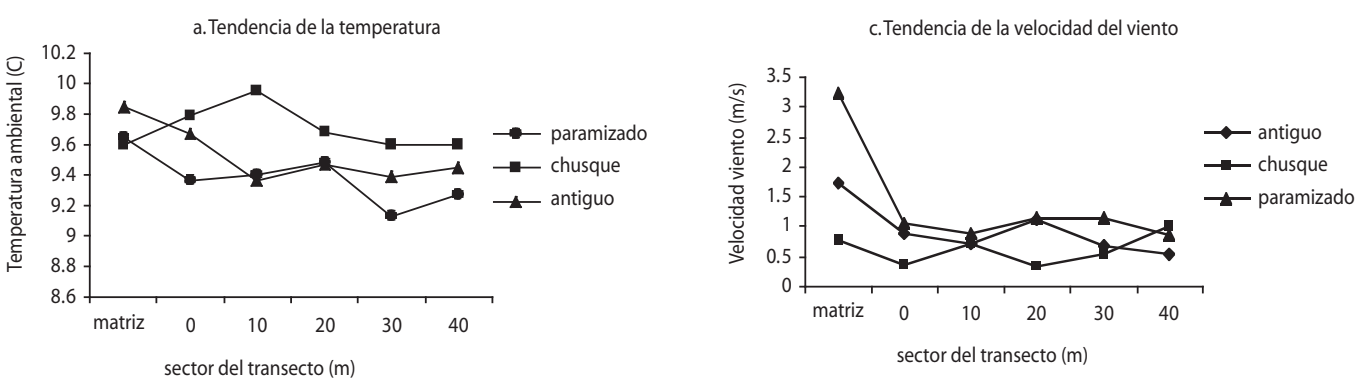

b. Tendencia de la humedad relativa

d. Tendencia de la radiación lumínica
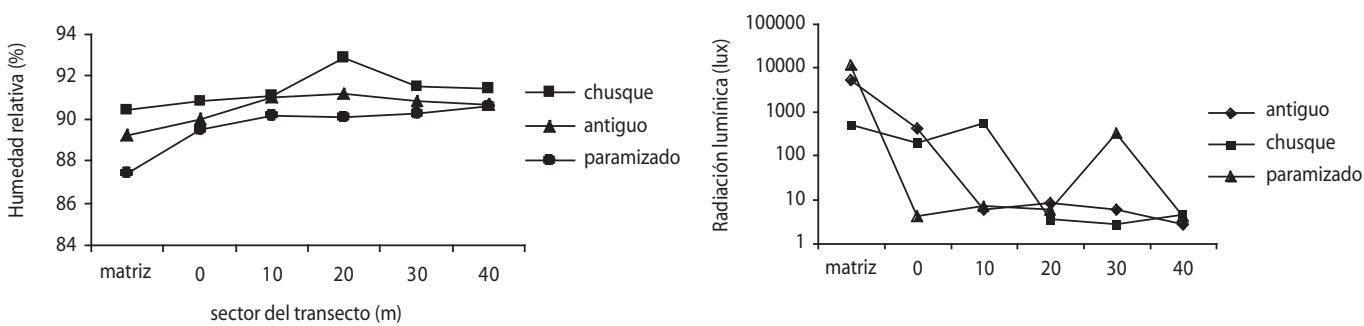

Fig. 3. Tendencias de las variables ambientales a lo largo del transecto matriz-interior de bosque según el tipo de borde.

Fig. 3. Tendencies of the environmental variables with distance in the transect matrix-interior of the forest for each edge type. 
bomplandianum, acompañado comúnmente por T. grossa, Gaiadendron punctatum y Miconia squamulosa (Cuadro 2). La matriz circundante presenta arbustos y parches de arbustos colonizadores (p.e. Gynoxis trianae y Hesperomeles goudotiana acompañadas por las especies de borde) cuya presencia amortigua el microclima dentro del bosque y los efectos de la matriz, por lo que la zona de borde antiguo sólo tiene 10 $\mathrm{m}$ de amplitud, en contraste con los otros tipos de borde donde oscila entre los $10 \mathrm{~m}$ y los 20 $\mathrm{m}$, como reveló el AC para la composición de especies que explicó el $85 \%$ de la varianza con dos ejes (Fig. 1). Tanto en el borde de chusque como en el antiguo, los disturbios principalmente por tala y extracción de madera y leña fueron moderados, resultando en la presencia de pocos claros unida a una estructura compleja con árboles que alcanzan los $10 \mathrm{~m}$ de altura en el borde y los $15 \mathrm{~m}$ en el interior; cabe resaltar que la abundancia relativa de estos árboles en el borde antiguo tiende a ser casi el doble que en el de chusque. Por su orientación en sotavento, el borde antiguo como el de chusque, se encuentra resguardado de la acción directa del viento y presenta condiciones microclimáticas intermedias entre los otros dos (chusque y paramizado) (Fig. 3, Anexo 2). La vegetación del borde antiguo posee la capacidad de autorregular el ambiente, amortiguando las condiciones drásticas de la matriz circundante.

3) Borde paramizado: Se ubica generalmente hacia la cima de las montañas, que es la zona más expuesta a los factores ambientales, además por su orientación en barlovento (SE) recibe la influencia directa del viento. El disturbio por explotación y extracción de madera fue muy intenso, la vegetación en su estructura carece de los árboles más altos encontrados en los otros tipos de borde; así en este sector los

CUADRO 2

Abundancia relativa de especies en cada tipo de borde

TABLE 2

Relative abundance of species in each edge type

$\begin{array}{lccc}\text { Especie } & \begin{array}{c}\text { Chusque } \\ \text { Abundancia (\%) }\end{array} & \begin{array}{c}\text { Antiguo } \\ \text { Abundancia (\%) }\end{array} & \begin{array}{c}\text { Paramizado } \\ \text { Abundancia (\%) }\end{array} \\ \text { Tibouchina grossa } & 24.0 & 35.4 & 14.0 \\ \text { Chusquea scandens } & 42.0 & <1 & <1 \\ \text { Gaiadendron punctatum } & - & 17.7 & 21.8 \\ \text { Macleania rupestris } & 2.0 & 2.0 & 18.0 \\ \text { Miconia ligustrina } & 14.3 & 5.7 & 0.7 \\ \text { Miconia squamulosa } & 4.0 & 11.3 & 2.1 \\ \text { Pentacalia pulchella } & 2.0 & 6.0 & 3.0 \\ \text { Weinmannia balbisiana } & 1.0 & 4.0 & 5.0 \\ \text { Drimys granadensis } & 3.0 & - & 6.8 \\ \text { Ugni myricoides } & 0.5 & 2.4 & 4.0 \\ \text { Ageratina } \text { sp. } & - & - & 5.0 \\ \text { Viburnum triphyllum } & 1.1 & 3.2 & 0.3 \\ \text { Weinmannia tomentosa } & - & 2.0 & 2.0 \\ \text { Hedyosmum bomplandianum } & 2.0 & 1.0 & 1.4 \\ \text { Clethra fimbriata } & - & - & 3.0 \\ \text { Myrsine dependens } & - & 2.0 & 0.4 \\ \text { Oreopanax bogotensis } & - & 0.8 & 1.0 \\ \text { Myrsine guianensis } & 1.1 & - & 0.7 \\ \text { Myrsine coriacea } & - & - & 1.0\end{array}$


árboles no superan los $8 \mathrm{~m}$ de altura y en el interior del bosque su altura es inferior a los $10 \mathrm{~m}$, con presencia de numerosos claros en el dosel y muchos árboles muertos en pie y caídos. Las especies más abundantes en la zona de borde son G. punctatum, Macleania rupestris, T. grossa, y Drimys granadensis (Cuadro 2); la segunda coloniza el sotobosque con una alta cobertura. La vegetación carece de la capacidad de amortiguar las condiciones ambientales, por lo que las variables evaluadas presentan un comportamiento extremo dentro del bosque, similar al de la matriz (Fig. 3, Anexo 2) cuando se comparan a lo largo del día; este tipo de borde tiende a ser el más frío y seco de los tres; su escasa capacidad de autorregulación conlleva a la muerte de los árboles del bosque y a la alta presencia de especies de páramo y/o subpáramo (Hypericum goyanesii, Calamagrostis effusa y Pernettya prostrata) capaces de tolerar condiciones ambientales drásticas tanto en el bosque como en la matriz circundante.

En general, 10 especies se presentan simultáneamente en los tres bordes aunque su abundancia relativa varía en cada uno (Cuadro 2) (p.e. T. grossa, G. punctatum, M. rupestris, M. ligustrina y $M$. squamulosa). Tres especies son exclusivas del borde paramizado (Ageratina sp., Clethra fimbriata y Myrsine coriacea) y el resto se encuentra principalmente en dos bordes a la vez. Cabe destacar la presencia en el borde, de especies típicamente consideradas como del bosque maduro o de interior, como $D$. granadensis, $H$. bomplandianum, Weinmannia tomentosa y $W$. balbisiana.

Forma y área del parche: Se encontró que un mismo tipo de borde puede encontrarse en fragmentos de bosque con tamaño y forma diferentes, por lo que no son características distintivas, e igualmente puede encontrarse más de un tipo de borde en un mismo parche.

\section{DISCUSIÓN}

Todos los parches de bosque estudiados fueron modelados por una historia de disturbios en mayor o menor grado antes de formar parte de la Reserva. Los parches de bosque menos alterados están aislados, poseen forma alargada y los pequeños casi circular, lo que contribuye a reducir los efectos de borde. Se encuentran en zonas de pendiente, generalmente poco accesibles y menos útiles para otros usos del suelo; estas condiciones pueden haberlos protegido de una mayor explotación y degradación por el hombre, como reportan estudios en otros bosques tropicales (Williams-Linera et al. 2002). El disturbio se considera como el principal determinante de la dinámica del bosque y la diversidad de árboles en escalas locales y regionales (Ramírez-Marcial et al. 2001). En este estudio el efecto del régimen de disturbios parece ser más trascendental en la dinámica de un parche y sus bordes que aspectos como el área del mismo o su aislamiento, este fenómeno ha sido reportado por otros trabajos que además realzan la importancia de la calidad del hábitat en términos de $\mathrm{pH}$, contenido de materia orgánica y de nitrógeno del suelo (Dupré y Ehrlén 2002).

Además, se sabe que el tipo e intensidad de disturbio puede alterar la estructura, la composición florística (Ramírez-Marcial et al. 2001, Onaindia et al. 2004) y la dinámica de la regeneración de los bosques tropicales tanto de tierras bajas como de montaña (RamírezMarcial et al. 2001), siendo posible apreciar estos efectos incluso muchos años después (Williams-Linera 1990, Harper y Macdonald 2002). Evidentemente los parches de bosque estudiados manifiestan estos cambios especialmente en la zona de borde, incluso las alteraciones en la regeneración pueden estar ocurriendo dada la ausencia de plántulas, ya que durante el año de estudio solamente fue posible encontrar siete plántulas de $H$. bomplandianum y dos de $M$. rupestris. Por otra parte, el régimen de disturbios resulta ser determinante en la alteración de las condiciones microambientales, especialmente si hay pérdida de estratos arbóreos (Williams-Linera 1990, Davies-Colley et al. 2000), caso del borde paramizado, donde la acción de factores fisiográficos como altitud, exposición directa al viento y ubicación en la cima de la montaña, pueden magnificar dicho 
efecto del disturbio, o en caso contrario pueden reducirlo como en el borde antiguo. El resultado de la combinación de estos factores y de los demás aspectos analizados genera un tipo de hábitat de borde determinado (antiguo, de chusque o paramizado) con una composición de especies particular.

Los parches con borde paramizado tuvieron extracción intensiva de madera y leña, evidenciada en la ausencia de árboles altos en el interior (con más de $10 \mathrm{~m}$ ) y en el borde (con más de $8 \mathrm{~m}$ ); la ausencia de estos árboles alteró el patrón de regeneración del dosel y permitió la colonización masiva de los claros principalmente por $M$. rupestris, presentando actualmente un dosel abierto conformado por árboles de porte inferior al encontrado en los parches de los otros tipos de borde. Como en los fragmentos de bosque los árboles altos del dosel son los encargados de controlar los gradientes de humedad y temperatura generados por la acción del sol y el viento (Bannerman 1998), la reducción de su cobertura y la pérdida de los estratos arbóreos conduce a alteraciones microclimáticas, que a su vez afectan la estructura de la vegetación tanto del dosel como del sotobosque (Williams-Linera 1990, DaviesColley et al. 2000), resultando en la pérdida de la capacidad autorreguladora de las condiciones ambientales bajo el dosel (Chen et al. 1999). Lo anterior confirma que el dosel de bajo porte del borde paramizado no es capaz de amortiguar las condiciones ambientales externas, más drásticas por su ubicación en barlovento. Otras evidencias de la poca autorregulación ambiental del borde paramizado se aprecian no sólo en las variables ambientales registradas dentro del bosque y en la presencia de árboles muertos en pie o caídos, cuyo número es alto en comparación con los otros tipos de borde; sino también, en la presencia en la matriz, de individuos propios del páramo y/o el subpáramo, siendo altamente contrastante con el bosque, lo cual limita la colonización de las especies del mismo, incapaces de tolerar sus condiciones rigurosas. Por todo lo anterior, este tipo de borde requiere la mayor intervención para lograr la recuperación del bosque, encaminada a promover la colonización de la matriz por especies como $T$. grossa, $W$. tomentosa, $W$. balbisiana, G. punctatum e incluso P. pulchella, que contribuyan a disminuir la influencia de la matriz sobre el bosque y a la vez generen condiciones más propicias para otras del borde en la RFMC con poca tolerancia a condiciones extremas como M. ligustrina, M. squamulosa y $H$. bomplandianum, favoreciendo a su vez la regulación del microambiente en el interior del bosque; esto permitiría a futuro el establecimiento de las especies sensibles de mayor porte típicas del bosque maduro, que sí se encuentran en otros sectores del mismo parche tales como O. bogotensis y Schefflera sp.

Los bordes antiguo y de chusque se asemejan en la presencia de árboles altos tanto en la zona de borde como en el interior del bosque, los cuales contribuyen a su autorregulación ambiental; además en ambos se presentan arbustos colonizadores de la matriz como $T$. grossa, M. ligustrina, M. squamulosa. Sin embargo la diferencia que puede haber conducido a diferentes tipos de borde parece radicar en el número de dichos árboles de porte medio $\mathrm{y}$ alto, siendo mayor en el borde antiguo. Estos árboles juegan un papel fundamental en la regeneración del bosque, pues sus copas tienden a extenderse lateralmente cerrando los claros derivados de la extracción de leña y madera, amortiguando las condiciones ambientales y favoreciendo la colonización por las especies del mismo parche; procesos similares se presentan en diferentes tipos de bosque incluso en diferentes latitudes (Forman 2001). Lo anterior unido a un mayor tiempo de abandono y al abrigo del viento, puede haber contribuido a la colonización de la matriz adyacente al borde antiguo, por especies pioneras arbustivas (como Bucquetia glutinosa y Ageratina gynoxoides) así como por aquellas del borde del bosque (como T. grossa y M. squamulosa) conduciendo a un estado avanzado de sucesión. La colonización por pioneras arbustivas puede facilitar la expansión del bosque e incluso contribuir a suavizar la influencia de la matriz sobre el mismo; lo anterior unido a la menor amplitud de este borde $(10 \mathrm{~m})$, revela la presencia de los 
procesos de regeneración del bosque en forma autónoma. Por consiguiente, este tipo de borde no requiere ningún tipo de intervención, pues el contraste con la matriz debe tender a reducirse con el tiempo, de acuerdo con lo planteado por Harper et al. (2005).

En contraste, en los parches con borde de chusque, la menor abundancia de árboles altos puede haber impedido que el dosel sellara por completo los claros, fruto de la acción antrópica, permitiendo un mayor ingreso de luz, que unido a la mayor humedad y posiblemente a la menor altitud favoreció el proceso de colonización del chusque en el sector de borde. La rápida expansión vegetativa del chusque formó una barrera protectora del viento y otras condiciones microclimáticas adversas, además de proteger al bosque del ingreso de propágulos de los pastizales. Sin embargo, también inhibe la colonización de la matriz por parte de las especies del bosque como T. grossa y M. ligustrina, las cuales logran establecerse tras la muerte periódica de la gramínea, pero perecen cuando ésta vuelve expandirse creciendo sobre ellas, sometiéndolas a una fuerte competencia por luz, nutrientes y espacio (Trujillo 2004). Este conjunto de circunstancias evita, en la matriz aledaña al borde de chusque, la formación de los parches de arbustos colonizadores presentes en la del borde antiguo, deteniendo el avance sucesional; pues se sabe que toda matriz a pesar de sus condiciones contrastantes y adversas tiende a ser colonizada por arbustos empezando por la zona más cercana al borde, debido a la influencia del ambiente del bosque y a mayor distancia de éste, la lluvia de semillas de las especies arbustivas disminuye (Díaz 2004). Por lo anterior, es indispensable controlar al chusque para permitir la colonización de la matriz y la formación de parches arbustivos que contribuyan a la expansión del bosque como sucede en el borde antiguo, aprovechando aquellas especies que prefieren este borde como $T$. grossa, M. ligustrina y M. squamulosa, cuya alta reproducción vegetativa resulta ventajosa en estas circunstancias.

\section{AGRADECIMIENTOS}

Este trabajo fue realizado gracias a la financiación de COLCIENCIAS, el apoyo de la alcaldía y la UMATA del municipio de Cogua, así como del personal de guardabosques y la Escuela de Restauración Ecológica de la Universidad Nacional de Colombia.

\section{RESUMEN}

Se caracterizaron tres tipos de borde de bosque altoandino: borde de Chusquea scandens, paramizado y antiguo; este último denominado así por presentar un estado sucesional más avanzado. Se seleccionaron dos parches de bosque por cada tipo de borde, para analizar 13 criterios entre fisiográficos, microclimáticos, estructura y composición de la vegetación. Tres de los criterios (pendiente, forma y área del parche) son poco determinantes del tipo de hábitat de borde, los demás están estrechamente relacionados con las condiciones del microambiente y éste a su vez con la estructura y composición de la vegetación, confiriendo características particulares a cada hábitat de borde. El borde paramizado muestra la menor capacidad de autorregulación ambiental estando más expuesto a la fluctuación en estas variables, debido a su exposición directa a la acción del viento y a la pérdida de los estratos arbóreos más altos (entre 10 y $15 \mathrm{~m}$ ), por lo que requiere la mayor intervención para su restauración. El borde de chusque es el más resguardado al actuar esta especie como una barrera protectora, que sin embargo debe ser controlada para promover la expansión del bosque como habita en el borde antiguo, el cual presenta una capacidad reguladora intermedia entre los otros dos.

Palabras clave: Chusquea scandens, bordes de bosque, disturbio, matriz, microclima, paramización, restauración ecológica.

\section{REFERENCIAS}

Absjornsen, H., M. Ashton, D. J. Vogt \& S. Palacios. 2004. Effects of habitat fragmentation on the buffering capacity of edge environments in a seasonally dry tropical oak forest ecosystem in Oaxaca, Mexico. Agr Ecosyst Environ. 103: 481-495.

Anderson, M. J. 2001. A new method for non-parametric multivariate analysis of variance. Austral Ecol. 26: $32-46$.

Arango, S. 2002. Edge effects on tree regeneration in the Colombian Andes. Tesis doctoral. Universidad de Missouri, Saint Louis, Missouri, EEUU. 231 p. 
Bannerman, S. 1998. Biodiversity and interior habitats: The need to minimize edge effects. Extension note 21. Ministry of forest Research program, British Columbia. In Biodiversity management concepts in Landscape Ecology. British Columbia, Vancouver, B.C., Canadá. 8 p.

Buckley L. B. 2000. The dynamics of temperate forest fragmentation: an examination of susceptibility to woody invasive species. Trabajo de grado, Williams College, Williamstown, Massachusetts, EEUU. 74 p.

Chen, J., S. Saunders, T. Crow, R. Naiman, K. Brosofske, G. Mroz, B. Y Brookshire \& J. Franklin. 1999. Microclimate in forest ecosystem and landscape ecology. Variations in local climate can be used to monitor and compare the effects of different management regimes. Bioscience 49: 288-297.

Davies-Colley, D., G. Payne \& M. Elswijk. 2000. Microclimate gradients across a forest edge. New Zealand. J. Ecol. 24: 111-121.

Díaz, R. 2004. Variación espacio-temporal de la lluvia de semillas en pastizales abandonados de alta montaña. Trabajo de grado, Departamento de Biología, Universidad Nacional, Bogotá, Colombia. 165p.

Didham, R. K. \& J. H. Lawton. 1999. Edge structure determines the magnitude of changes in microclimate and vegetation structure in tropical forest fragments. Biotropica 31: 17-30.

Dupré, C. \& J. Ehrlén. 2002. Habitat configuration, species traits and plant distributions. J. Ecol. 90: 796-805.

Forman, R. T. 2001. Land Mosaics. The ecology of landscapes and regions. Universidad de Cambridge, Londres, Inglaterra. 632 p.

Fox, B.J., J. Taylor, M. D. Fox \& C. Williams. 1997. Vegetation changes across edges of rainforest remnants. Biol Conserv. 82: 1-13.

García, N. \& C. Giraldo. 2003. Caracterización florística de la Reserva Forestal Municipal de Cogua, Cundinamarca. Informe final. UMATA, Santiago de Calí, Valle del Cauca, Colombia. 286 p.

Hammer Ø., D.A.T. Harper \& P.D. Ryan, 2001. PAST: Palaeontological Statistics software package for education and data analysis. Paleontología electrónica 4: 9 pp. PAST version 1.40 Øyvind Hammer \& D.A.T. Harper (enero 2006).

Harper, K.A. \& S.E. Macdonald. 2002. Structure and composition of edges next to regenerating clear-cuts in mixed-wood boreal forest. J. Veg Sci. 13: 535-546.

Harper, K.A., S.E. Macdonald, P. Burton, J. Chen, K. Brosofske, S. Saunders, E. Euskirchen, D. Roberts,
M. Jaiteh \& P. Esseen. 2005. Edge influence on forest structure and composition in fragmented landscapes. Conserv Biol. 19: 1-15.

Leon, O. 2002. Distribución espacial de comunidades vegetales de páramo en un gradiente altitudinal con vegetación natural y en proceso de paramización (Reserva Forestal del Municipio de Cogua, Cundinamarca). Trabajo de grado. Departamento de Ecología. Universidad Javeriana, Bogotá, Colombia. 148 p.

Mesquita, R. C., P. Delamonica \& W. Laurance. 1999. Effect of surrounding vegetation on edge-related tree mortality in amazonian forest fragments. Biol Conserv. 91: 129-134.

Murcia, C. 1995. Edge effects in fragmented forest: implications for conservation. Tree 10: 58-62.

Onaindia M., J. Dominguez, I. Albizu, G. Garbisu \& I. Amezaga. 2004. Vegetation diversity and vertical structure as indicators of forest disturbance. Forest Ecol Manag. 195: 341-354.

Primack, J. 1993. Conservation Biology. Sinauer Associates. Sunderland, Massachusetts, EEUU. 277 p.

Ramírez-Marcial, N., M. González-Espinosa \& G. Williams-Linera. 2001. Anthropogenic disturbance and tree diversity in montane rain forest in Chiapas, Mexico. Forest Ecol Manag. 154: 311-326.

Trujillo, L. 2004. Caracterización de bordes con Chusquea scandens y evaluación de la regeneración luego de disturbios experimentales. Trabajo de grado, Departamento de Biología, Universidad Nacional, Bogotá, Colombia. 125 p.

Williams-Linera, G. 1990. Vegetation structure and environmental conditions of forest edges in Panama. J. Ecol. 78: 356-373.

Williams-Linera, G., V. Dominguez-Gastelú \& M. GarcíaZurita. 1998. Microenvironment and floristics of different edges in a fragmented tropical rainforest. Conserv Biol. 12: 1091-1102.

Williams-Linera, G., R. H. Manson \& E. Isunza. 2002. La fragmentación del bosque mesófilo de montaña y patrones de uso del suelo en la región oeste de Xalapa, Veracruz, México. Madera Bosques 8: 73-89.

Zambrano, M. A. 1995. Plan de manejo del área de Reserva Forestal Protectora de los nacimientos de quebradas Honda y Calderitas en el municipio de Cogua. Corporación Autónoma Regional de Cundinamarca (CAR). Subdirección de operaciones de áreas protegidas, Bogotá, Colombia. 327 p. 


\section{ANEXO 1}

ANOVA (PERMANOVA v. 1.6) para los tipos de borde y sectores de los transectos realizados en el bosque

\section{APPENDIX 1}

ANOVA (PERMANOVA v. 1.6) for edge types and sectors of transects into the forest

\section{Experimental Design}

Factor 1 is borde

Factor 2 is distance

The sample size (n) with 3 levels and is fixed

with 5 levels and is fixed

$$
=2
$$

The total no. of observations $=30$

The total no. of variables $=45$

Factor 2 is nested in factor 1

Results. Permutational Multivariate Analysis of Variance

$\begin{array}{lcccccc}\text { Source } & \text { df } & \text { SS } & \text { MS } & \text { F } & \text { P(perm) } & \text { P(MC) } \\ \text { bo } & 2 & 15998.54 & 7999.2702 & 3.6446 & 0.0010 & 0.0020 \\ \text { di(bo) } & 12 & 39617.09 & 3301.4243 & 1.5042 & 0.0160 & 0.0210 \\ \text { Residual } & 15 & 32922.80 & 2194.8533 & & & \\ \text { Total } & 29 & 88538.43 & & & & \end{array}$

No transformation, No standardization

Analysis based on Bray-Curtis dissimilarities

Unrestricted permutation of raw data using correct permutable units

Integer used as seed $=5$, No. of permutations used $=999$

\section{Pair-wise a posteriori comparisons}

Name of the factor being tested: distance

No. of sets of pairwise comparisons $=3$

No. of groups compared within each set $=5$

Total no. of tests done $=30$

Integer chosen for the random seed $=6$

\begin{abstract}
No. of raw observations per group $=2$
No. of permutable units per group $=2$

No. of permutations done $=999$

Permutation of raw data using appropriate permutable units
\end{abstract}

Tests among levels of distance within: level 1 of Borde

$\begin{array}{ccccc}\text { Groups } & \mathrm{t} & \text { P_perm } & \text { P_MC } & \text { \#unique vals } \\ (1,2) & 0.8043 & 1.0000 & 0.6250 & 3 \\ (1,3) & 0.4274 & 1.0000 & 0.8320 & 3 \\ (1,4) & 0.8360 & 0.6670 & 0.5260 & 3 \\ (1,5) & 1.3874 & 0.3270 & 0.2520 & 3 \\ (2,3) & 0.8499 & 1.0000 & 0.5290 & 3 \\ (2,4) & 1.0289 & 0.3230 & 0.4180 & 3 \\ (2,5) & 1.2786 & 0.3300 & 0.2460 & 3 \\ (3,4) & 0.6929 & 1.0000 & 0.7000 & 3 \\ (3,5) & 1.3076 & 0.3420 & 0.2550 & 3 \\ (4,5) & 1.5154 & 0.3410 & 0.1900 & 3\end{array}$

Average dissimilarities within/between groups

$\begin{array}{cccccc}1 & 2 & 3 & 4 & 5 & \\ 1 & 70.24 & & & & \\ 2 & 55.98 & 52.212 & & & \\ 3 & 56.85 & 62.188 & 82.301 & & \\ 4 & 60.12 & 58.987 & 62.973 & 65.657 & \\ 5 & 93.83 & 80.293 & 97.054 & 97.117 & 84.507\end{array}$

Resultados similares se obtienen para la distancia en los otros dos niveles del factor borde. 
ANEXO 2

Variables ambientales a lo largo del transecto matriz-interior de bosque según el tipo de borde

APPENDIX 2

Environmental variables with distance in the transect matrix-interior of the forest for each edge type

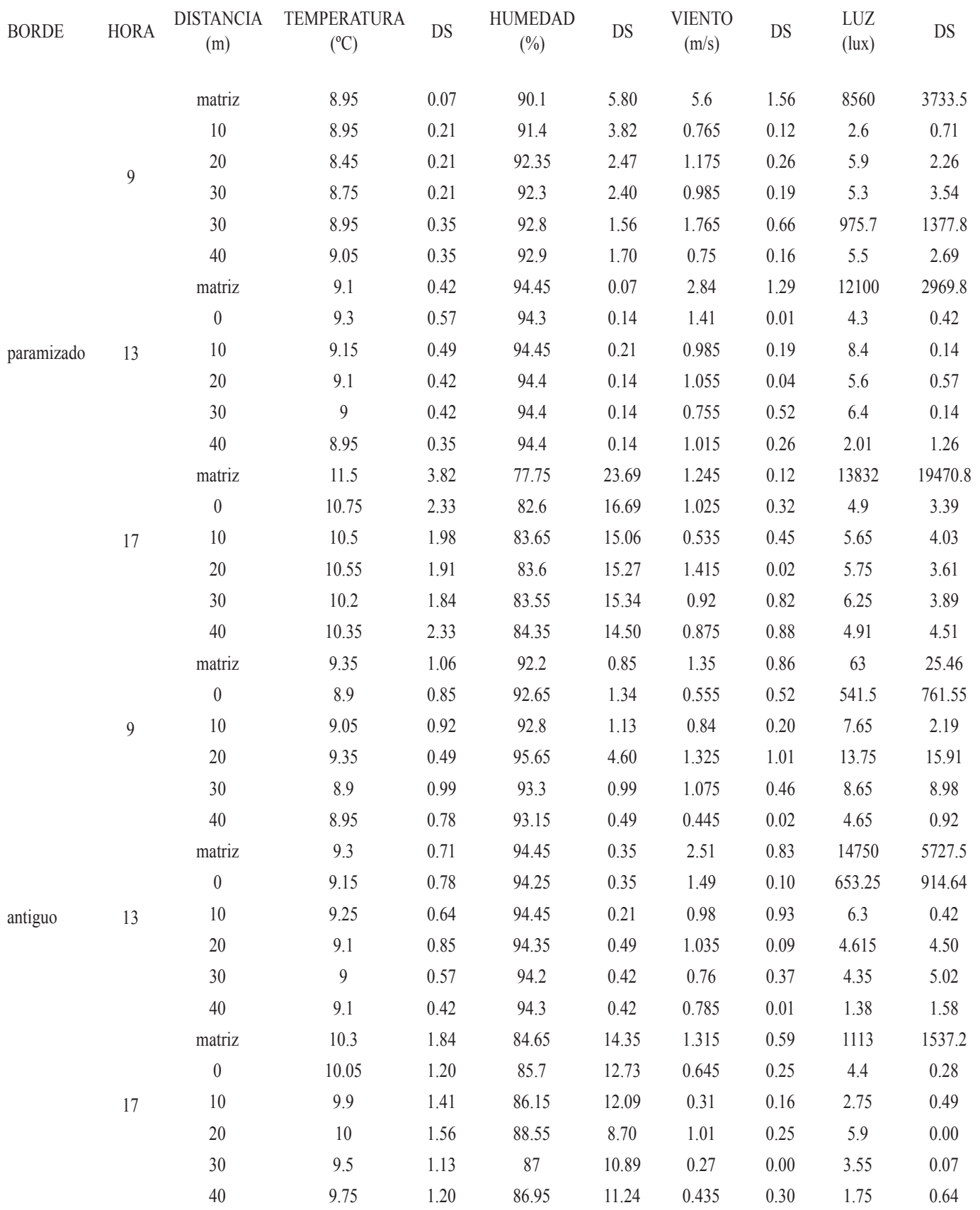




\begin{tabular}{|c|c|c|c|c|c|c|c|c|c|c|}
\hline BORDE & HORA & $\begin{array}{l}\text { DISTANCIA } \\
(\mathrm{m})\end{array}$ & $\begin{array}{c}\text { TEMPERATURA } \\
\left({ }^{\circ} \mathrm{C}\right)\end{array}$ & DS & $\begin{array}{c}\text { HUMEDAD } \\
(\%)\end{array}$ & DS & $\begin{array}{l}\text { VIENTO } \\
(\mathrm{m} / \mathrm{s})\end{array}$ & DS & $\begin{array}{l}\text { LUZ } \\
\text { (lux) }\end{array}$ & DS \\
\hline \multirow{18}{*}{ chusque } & \multirow{7}{*}{9} & matriz & 9.7 & 1.13 & 88.55 & 7.14 & 1.04 & 0.74 & 48.5 & 4.95 \\
\hline & & 0 & 9.1 & 0.00 & 89.15 & 6.72 & 0.33 & 0.17 & 5.3 & 3.25 \\
\hline & & 10 & 9.4 & 0.42 & 90.4 & 5.23 & 1.07 & 0.14 & 833.05 & 1169.4 \\
\hline & & 20 & 9.35 & 0.35 & 90.55 & 5.02 & 0.295 & 0.13 & 4.45 & 2.76 \\
\hline & & 30 & 9.6 & 0.99 & 90.7 & 4.53 & 0.78 & 0.52 & 2.35 & 0.07 \\
\hline & & 40 & 9.5 & 0.57 & 91.05 & 3.89 & 0.95 & 0.59 & 7.3 & 2.83 \\
\hline & & matriz & 9.7 & 0.85 & 94.3 & 0.57 & 0.87 & 0.17 & 69 & 29.70 \\
\hline & \multirow{5}{*}{13} & 0 & 10.1 & 0.99 & 94.2 & 0.28 & 0.295 & 0.06 & 559.5 & 679.53 \\
\hline & & 10 & 10.15 & 0.64 & 94.1 & 0.00 & 0.68 & 0.04 & 700.59 & 989.12 \\
\hline & & 20 & 9.15 & 1.06 & 94 & 0.14 & 0.345 & 0.08 & 4.19 & 4.26 \\
\hline & & 30 & 9.15 & 0.64 & 93.95 & 0.21 & 0.525 & 0.25 & 4.1 & 1.27 \\
\hline & & 40 & 9.1 & 0.85 & 93.9 & 0.28 & 1.4 & 0.14 & 4.64 & 5.03 \\
\hline & \multirow{6}{*}{17} & matriz & 9.4 & 0.00 & 84.7 & 14.85 & 0.44 & 0.33 & 1332.05 & 1878.00 \\
\hline & & 0 & 10.15 & 0.49 & 86.55 & 11.53 & 0.505 & 0.39 & 4.45 & 3.75 \\
\hline & & 10 & 10.3 & 0.71 & 88.65 & 8.56 & 0.445 & 0.11 & 1.55 & 1.48 \\
\hline & & 20 & 10.55 & 1.06 & 89.05 & 7.85 & 0.385 & 0.26 & 1.35 & 0.21 \\
\hline & & 30 & 10.05 & 1.77 & 87.95 & 9.69 & 0.305 & 0.25 & 1.6 & 1.56 \\
\hline & & 40 & 10.2 & 1.27 & 87.15 & 11.10 & 0.65 & 0.49 & 1.1 & 0.85 \\
\hline
\end{tabular}

